\title{
GLAD!
}

Revue sur le langage, le genre, les sexualités

$06 \mid 2019$

Varia

\section{Introduction \#6}

Issue $n^{\circ}$ : Introduction

Julie Abbou, Aron Arnold, Sophie Bailly, Maria Candea, Mona GérardinLaverge, Magali Guaresi et Noémie Marignier

\section{(2) OpenEdition}

\section{Journals}

Édition électronique

URL : http://journals.openedition.org/glad/1583

DOI : $10.4000 /$ glad. 1583

ISSN : 2551-0819

\section{Éditeur}

Association GSL

\section{Référence électronique}

Julie Abbou, Aron Arnold, Sophie Bailly, Maria Candea, Mona Gérardin-Laverge, Magali Guaresi et Noémie Marignier, « Introduction \#6 », GLAD! [En ligne], 06 | 2019, mis en ligne le 01 juillet 2019 consulté le 17 décembre 2020. URL : http://journals.openedition.org/glad/1583 ; DOI : https://doi.org/ 10.4000/glad. 1583

Ce document a été généré automatiquement le 17 décembre 2020.

\section{c) (i)}

La revue GLAD! est mise à disposition selon les termes de la Licence Creative Commons Attribution -

Pas d'Utilisation Commerciale - Pas de Modification 4.0 International. 


\title{
Introduction \#6
}

\author{
Issue $n^{\circ} 6$ : Introduction
}

\section{Julie Abbou, Aron Arnold, Sophie Bailly, Maria Candea, Mona Gérardin- Laverge, Magali Guaresi et Noémie Marignier}

1 Ce sixième numéro de GLAD! est un numéro varia qui présente une grande cohérence thématique: dans la rubrique "Recherches", les articles explorent des formes de résistance artistique et culturelle féministe et/ou queer, dans différents contextes (Amérique du Nord, Amérique Latine, Europe, Afrique) et par le biais de divers moyens d'expression (cinéma, littérature, arts plastiques). La rubrique "Créations», qui propose deux œuvres littéraires originales jouant de façon féministe et subversive avec et sur la langue, fait écho de très belle façon à ces articles scientifiques. La rubrique "Actualités » s'enrichit de plusieurs recensions et résumés de thèse qui attestent de la variété et du dynamisme des études francophones en genre et langage. Enfin, notre «Chronique » met en évidence la dimension politique de ces études en discutant la question du changement de prénom.

2 En « Recherches ", l'article de Julie Lavigne, Myriam Le Blanc Élie et Sabrina Maiorano étudie un important corpus de films pornographiques féministes et/ou queer. Les autrices démontrent que ces productions constituent de véritables formes de résistance artistique et culturelle aux scripts sexuels hétéronormatifs traditionnels, surtout en ce qui concerne la représentation de l'agentivité sexuelle des femmes et des personnes trans. Elles analysent par exemple la manière dont l'expression du consentement est mise en scène dans ces films. Leur article constitue une contribution importante aux études pornographiques, et élabore d'intéressants outils d'analyse féministe de l'agentivité sexuelle dans les productions cinématographiques.

L'article de Luc Schicharin explore lui aussi des formes de contre-récits artistiques, qui viennent contredire et bousculer les récits culturels traditionnels en mettant au jour leur hétéro-cisnormativité. Il propose une lecture particulièrement intéressante et ambitieuse des œuvres d'artistes trans, Yishay Garbasz, Wynne Neilly, Kris Grey et Cassils, dont il démontre qu'elles viennent déconstruire la vision cisnormative du temps, dans ses différentes dimensions: comme temporalité de l'espèce humaine (évolutionnisme darwinien) et comme temporalité de l'individue (évolution du corps 
en fonction des différents âges). Luc Schicharin montre que les œuvres de ces artistes trans mettent en question cette vision linéaire et évolutionniste, et ouvrent d'autres manières de penser le temps, d'autres temporalités corporelles.

4 En ce qui concerne l'article de Didier Brou Anoh, celui-ci interroge le pouvoir politique des discours littéraires à travers l'analyse de deux romans qui traitent de la tragédie du génocide rwandais. L'auteur défend l'idée que le langage véhément de révolte employé par V. Tadjo dans L'ombre d'Imana. Voyage jusqu'au bout du Rwanda et par M. Ilboudo dans Murekatété parvient à forger et faire entendre un féminisme africain révolutionnaire, capable à la fois de mettre en mots le chaos et de construire l'espoir. Son analyse met en miroir, en prenant appui sur des extraits des deux romans, deux faces du discours génocidaire : la dénonciation - qui flirte avec les limites de l'indicible des crimes de masse - et, ce qui semble à peine pensable dans ce contexte : une forme d'optimisme.

Suivant notre ligne éditoriale de publications de textes venus d'ailleurs, ce numéro est l'occasion de découvrir, grâce à la formidable traduction de Marion Marchetti, le travail de Maria Jesus Fariña Busto, de l'université de Vigo en Espagne. Son étude porte sur la déconstruction des injonctions de genre dans l'œuvre de cinq écrivaines de différentes époques et régions de l'Amérique hispanophone. Des extraits de textes de divers genres dont la poésie illustrent les stratégies de résistance féminine et féministe face aux contraintes et au silence que le patriarcat cherche à imposer dans le contexte hispanoaméricain. Quatre axes de résistance à l'imposition des rôles genrés sont explorés transversalement : la désobéissance, la négation, la subversion, la transgression. Pour Maria Jesus Fariña Busto ces œuvres proposent des modèles de réflexivité et de combat qui invitent à franchir les frontières et à démanteler les injonctions.

En «Créations », nous avons le plaisir de publier deux œuvres littéraires d'autrices qui travaillent la langue dans une perspective féministe et subversive. Typhaine D., autrice, comédienne, conférencière et militante, invente une langue féministe et une grammaire "Féminine Universelle", qui prend l'exact contrepied du «Masculin Universel » qui régit la langue française depuis son imposition aux $16^{\mathrm{e}}$ et $17^{\mathrm{e}}$ siècles. Dans «La Pérille Mortelle » que nous publions, Typhaine D. parodie les oppositions de l'Académie française à l'écriture inclusive, dont elle montre l'absurdité en proposant une simple, mais éloquente, inversion de la situation: les académiciens sont des académiciennes, la féminine l'emporte, et les noms de métier prestigieux n'existent pas au masculin. Ce texte, piquant et drôle, constitue un outil pédagogique et politique de premier ordre en faveur de l'écriture inclusive, et donne envie d'explorer la langue.

7 C'est dans la même veine audacieuse par rapport à la matérialité morphologique de la langue française que s'inscrivent les deux récits de Julie Sorel, rédigés dans un style qui rappelle les contes pour enfants ou la légende héritée de la nuit des temps et racontée au coin du feu. Cette autrice se présente comme la demi-sœur de Julien Sorel, issue d'un mariage secret, tardif et posthume entre Stendhal et Agatha Christie, ce qui nous donne déjà le ton. Son premier texte, intitulé « Roselyne et Roselon », aborde la transmission des noms entre époux sur un ton mi-philosophique mi-parodique, tandis que le second, intitulé "Mutation", revisite avec une forme décapante d'humour agenre le récit biblique de la Genèse.

8 Dans un style nettement plus sérieux, la chronique de Daniel Elmiger traite, elle aussi, de la question du choix des noms (plus exactement des prénoms) donnés aux êtres humains. Le texte interroge les liens entre l'imposition d'un prénom à la naissance et l'assignation d'un pronom, d'une identité de genre, pour défendre vigoureusement le 
droit à l'autodétermination, et davantage de flexibilité dans les procédures de changement de prénom.

Enfin, dans la rubrique "Actualités », une série de recensions et de résumés de thèse donnent à voir différentes facettes du dynamisme scientifique actuel des études en genre et langage. On y trouve des présentations critiques de deux ouvrages personnels : Luca Greco, Dans les coulisses du genre : la fabrique de soi chez les Drag Kings paru en 2018 et Denis Provencher, Queer Maghrebi French, paru en 2017. On y trouve également les présentations de deux ouvrages collectifs : le premier a été publié par le «laboratoire junior » GenERe (ENS de Lyon) et porte sur les épistémologies du genre (Épistémologies du genre. Croisements des disciplines, intersections des rapports de domination paru en 2018) et le second, Guide pédagogique Égalité entre les femmes et les hommes, la lutte contre les stéréotypes de genre a été réalisé par Sandra Tomc et dix enseignantes de Français Langue Étrangère pour aider les formateurs et formatrices de FLE à intégrer une perspective genre dans leur enseignement. La rubrique inclut également deux résumés de thèses récemment soutenues, à savoir : celle de Mona Gérardin-Laverge, qui croise la philosophie du langage et les études de genre pour étudier la construction du genre et les usages féministes du langage, et la thèse de Nicholas LoVecchio qui propose une étude historique et comparative des emprunts linguistiques et des internationalismes dans le lexique des homosexualités.

\section{RÉSUMÉS}

Ce sixième numéro de GLAD! est un numéro varia qui présente une grande cohérence thématique: dans la rubrique «Recherches", les articles explorent des formes de résistance artistique et culturelle féministe et/ou queer, dans différents contextes (Amérique du Nord, Amérique Latine, Europe, Afrique) et par le biais de divers moyens d'expression (cinéma, littérature, arts plastiques). La rubrique «Créations», qui propose trois œuvres littéraires originales jouant de façon féministe et subversive avec et sur la langue, fait écho de très belle façon à ces articles scientifiques. La rubrique « Actualités " s'enrichit de plusieurs recensions et résumés de thèse qui attestent de la variété et du dynamisme des études francophones en genre et langage, et la rubrique «Chronique » met en évidence la dimension politique de ces études en discutant la question du changement de prénom.

This sixth issue of GLAD! is a varia issue with a strong topical coherence: in the "Research" section, the articles explore forms of feminist and queer artistic and cultural resistance in different contexts (North America, Latin America, Europe, Africa) involving various means of expression (cinema, literature, visual arts). The "Creations" section, which provides two original literary works, playing in a feminist and subversive way with and about the language, echoes these scientific articles in a beautiful way. The "News" section is enriched with several reviews and thesis summaries that illustrate the variety and dynamism of French gender and language studies, and the "Chronicle" section highlights the political dimension of these studies by discussing the issue of the practice of changing first names. 


\section{INDEX}

Keywords : language, art, creation, gender, feminism

Mots-clés : langage, art, création, genre, féminisme

\section{AUTEURS}

\section{JULIE ABBOU}

Titulaire d'un doctorat de Sciences du Langage (Aix-Marseille Université) sur les modifications du genre linguistique pour des motifs politiques, Julie Abbou mène des recherches sur les apports théoriques réciproques des études de genre et des sciences du langage. Elle travaille également sur les dimensions sémiotiques du genre grammatical, ainsi qu'en rhétorique sur le traitement du genre dans différents types de discours. Récemment, elle a co-dirigé l'ouvrage Gender, Language and the Periphery. Grammatical and social gender from the margins (John Benjamins) et publié des articles dans des revues telles que Semen, Mots les langages du politique, Current issues in Language Planning, etc.

\section{ARON ARNOLD}

Aron Arnold est docteur de l'Université Sorbonne Nouvelle et actuellement chercheur postdoctoral à l'Université catholique de Louvain. Spécialiste en sociophonétique, il s'intéresse aux liens entre voix, genre et sexualité. Ses travaux portent sur le rôle de la voix dans la perception du genre, sur la manière dont la voix contribue à reproduire une idéologie de genre binaire, ainsi que sur le rôle de la voix dans la séduction.

\section{SOPHIE BAILLY}

Sophie Bailly est professeure des universités en sciences du langage à l'université de Lorraine, et membre de l'UMR 7118 ATILF CNRS dans l'équipe Didactique des Langues et Sociolinguistique. Elle est l'auteure de Les hommes les femmes et la communication : mais que vient faire le sexe dans la langue ?, monographie parue chez L'Harmattan en 2008. En parallèle de ses travaux sur la (re)production discursive des stéréotypes de genre, elle mène des recherches sur l'autonomisation dans l'enseignement et l'apprentissage des langues.

\section{MARIA CANDEA}

Maria Candea, sociolinguiste et sociophonéticienne, s'intéresse principalement au français contemporain parlé en France et aux idéologies linguistiques. Maitresse de conférences à l'Université Paris 3 Sorbonne nouvelle.

\section{MONA GÉRARDIN-LAVERGE}

Mona Gérardin-Laverge est docteure en Philosophie à l'Université Paris 1 Panthéon Sorbonne. Elle a soutenu en 2018 une thèse de philosophie intitulée Le langage est un lieu de lutte. La performativité du langage ordinaire dans la construction du genre et les luttes féministes. Elle est membre de l'Institut des Sciences Juridique et Philosophique de la Sorbonne.

\section{MAGALI GUARESI}

Magali Guaresi est docteure en histoire contemporaine de l'Université Côte d'Azur et postdocorante à l'Université Libre de Bruxelles. Elle est l'auteure de Parler au féminin. Les professions de foi des député.e.s sous la Cinquième République (1958-20007), monographie parue chez 
L'Harmattan en 2018. En parallèle de ses travaux sur le genre dans le discours politique, elle mène des recherches sur les représentations de l'islam dans le discours de presse francophone belge en collaboration avec le ReSIC (Université Libre de Bruxelles).

\section{NOÉMIE MARIGNIER}

Noémie Marignier est docteure en Sciences du Langage et Attachée Temporaire d'Enseignement et de Recherche à l'Université Paris 3 Sorbonne Nouvelle. Ses travaux de recherche portent sur l'articulation entre corps/sexe/sexualité et discours. Elle a soutenu en 2016 une thèse en analyse du discours intitulée Les matérialités discursives du sexe. La construction et la déstabilisation des évidences du genre dans les discours sur les sexes atypiques. Elle est membre associée du laboratoire Clesthia (Université Paris 3 Sorbonne Nouvelle) ainsi que de Pléiade (Université Paris 13 Sorbonne Cité). 